\title{
Distances to Planetary Nebulae
}

\author{
Arsen R. Hajian ${ }^{1}$ \\ ${ }^{1}$ United States Naval Observatory, 3450 Massachusetts Av, NW, Washington, DC 20392, USA \\ email: hajian@usno.navy.mil.
}

\begin{abstract}
In this talk, I describe methods for direct distance estimation to Planetary Nebulae $(\mathrm{PNe})$. I review the foremost of these methods, the Expansion Parallax Distance, and present some recent results from this technique. In addition, I will discuss the major contributions to the systematic and random distance errors resulting from the method (including ionization front motions and deviations from spherical target morphologies), and show preliminary evidence quantifying these effects.
\end{abstract}

Keywords. planetary nebulae: general, stars: distances

\section{Introduction}

Distances to galactic planetary nebulae are poorly-defined, even by the already loose standards of astronomical distance estimation. The most common statistical method for PNe distance estimation is the Shklovsky method (Shklovsky 1956). This technique infers a distance based on the assumption of a constant ionized mass. However, since the progenitors to $\mathrm{PNe}$ range in mass from $0.8-8 \mathrm{M}_{\odot}$, the Shklovsky distance is inherently uncertain by more than a factor of 2 , rendering it almost useless for individual objects. Despite this limitation, the Shklovsky distance has been a very useful estimator for investigating the general properties of PNe. The wide range of derived statistical distances is illustrated in Table 1 from Terzian \& Teymourian (2005), which shows various statistical distances for a sample of well-observed PNe. As seen in the Table, it is common to see distance variations from method to method in excess of 2 , even for nearby objects.

Recent efforts to determine precise distances to PNe are approaching fruition. Despite the increase in computational and observational cost over the Shklovsky approach, these techniques have the potential of reducing the distance uncertainties by an order of magnitude. Here, I review the two most common precision distance techniques for PNe: the trigonometric parallax and the expansion parallax distances.

\section{Trigonometric Parallax}

The simplest geometrical method, and one of the first techniques learned by astronomy students, is the trigonometric parallax. However, PNe are not conducive to trigonometric parallax distance estimation. Canonical PNe distances are $>1 \mathrm{kpc}$, and the parallax at this range is very small ( $<1$ mas), and out of range for most astrometric systems.

However, using a dedicated astrometric telescope, lots of observing time, and careful attention to data reduction procedures (especially differential refraction), scientists at the US Naval Observatory in Flagstaff, Arizona have achieved remarkable success measuring groundbased parallaxes. Currently, they have presented more than a dozen parallaxes to the central stars of PNe and with median parallax errors $<0.5$ mas. To date, twelve PNe with fractional parallax errors less than 20\% (Harris et al. 2006; Harris, this conference) have been measured. With additional observing in the next few years for these and some 


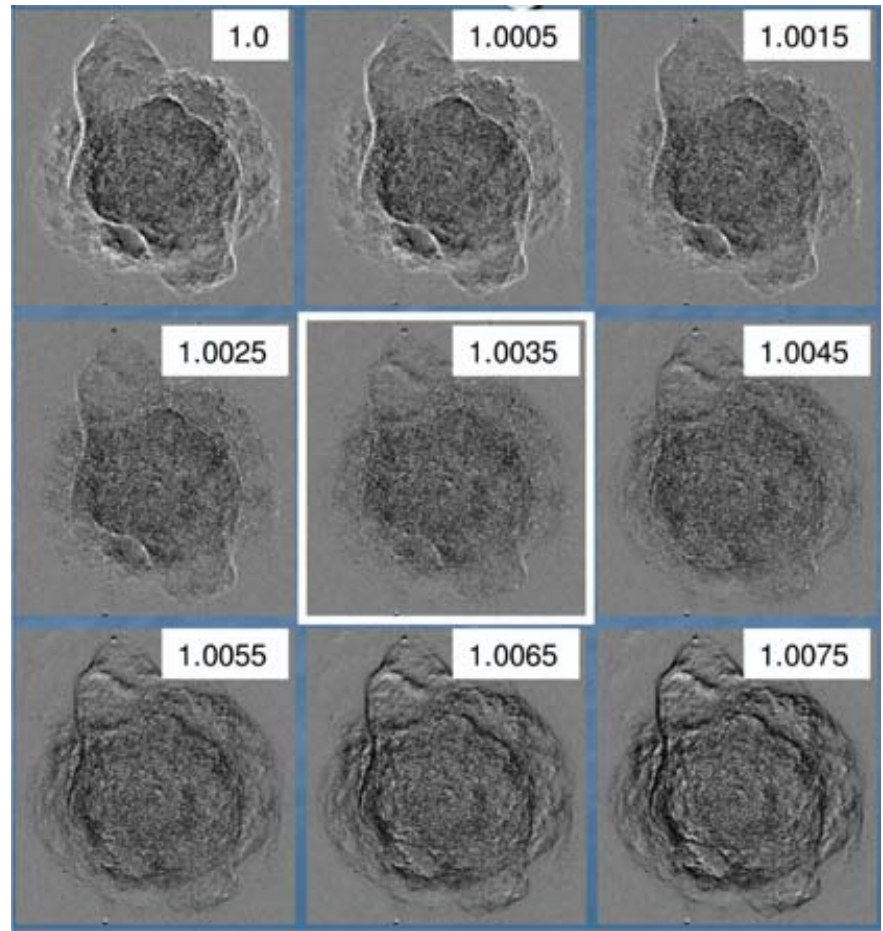

Figure 1. The magnification method as applied to NGC 3918. Shown are the residuals images resulting from the epoch 2 image minus a version of the epoch 1 image magnified by $M$. As can be seen, the residuals are minimized for $M=1.0035$.

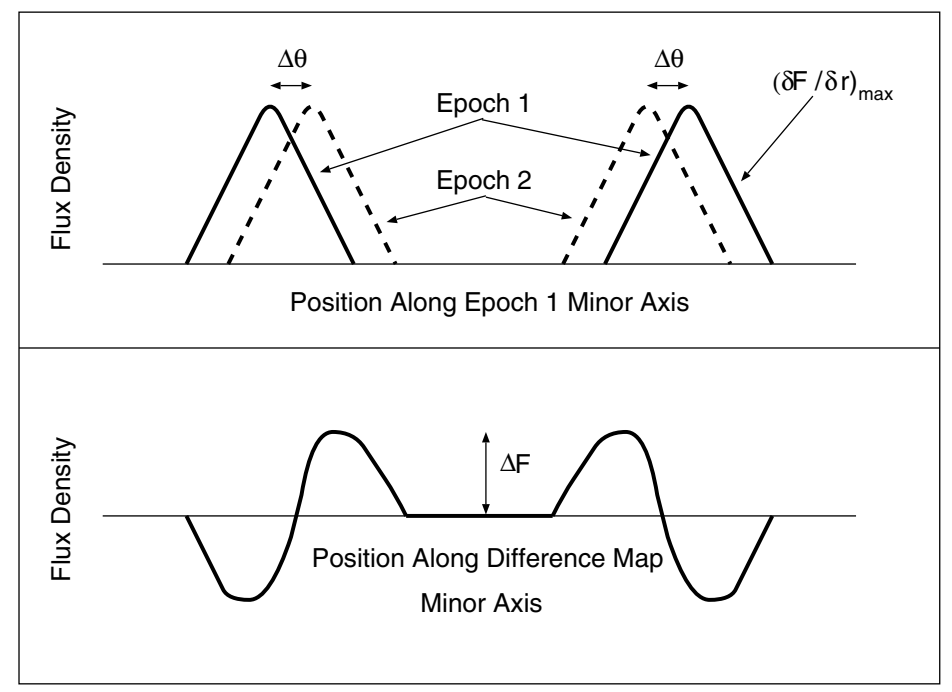

Figure 2. A schematic of the gradient technique for angular expansion measurement.

other relatively nearby $\mathrm{PNe}$, it will be possible to obtain distances to two dozen PNe with precisions better than $20 \%$ for comparison to other distance methods/estimators (Harris, 2006, private communication).

In the case of space-based parallaxes, the systematic error limit of the Hipparcos satellite is $\approx 1$ mas. The systematic floor conspires with the magnitude limit of Hipparcos to 


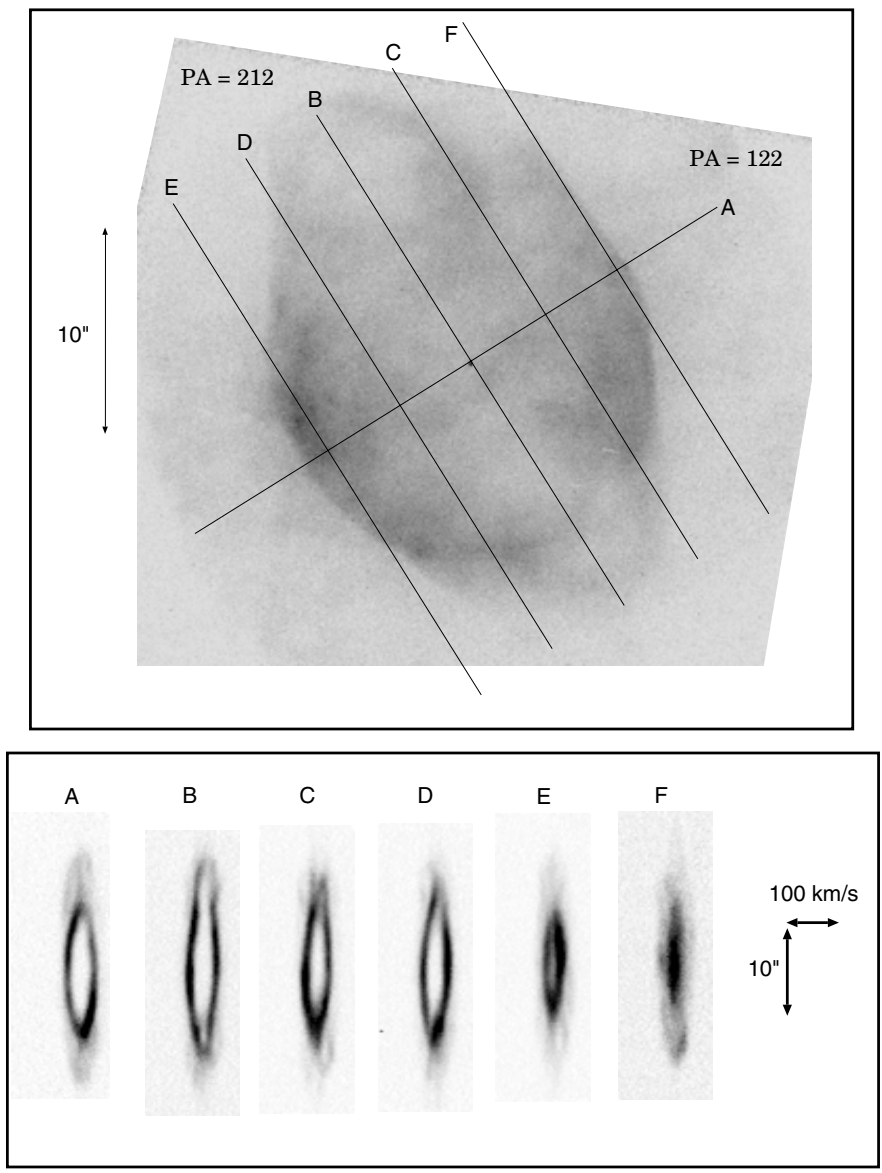

Figure 3. The data from NGC 7354 in the light of [O III]. The top figure shows the optical morphology with the slit positions denoted by thin lines labeled A-F. The position-velocity distributions resulting from the longslit spectra are shown at the bottom of the figure.

make candidates rare. To make matters worse, the small sample of PNe central stars (19) bright enough to be detectable and with parallaxes at least as large as this error floor gives problematic distances (Pottasch \& Acker 1998). A program currently underway with HST is measuring trigonometric parallaxes for four objects in common with the groundbased work, seeking to reach the highest accuracy currently possible. (Benedict et al. 2003 give a preliminary result.)

A major step forward in PNe parallax determination will arrive with the GAIA astrometric mapping satellite. Currently scheduled to launch in 2011, the GAIA mission is designed to be complete to $\mathrm{V}=20$, and to have an astrometric accuracy of $20-25 \mu$ as at $\mathrm{V}=15$. For comparison, there will be $\approx 50-70$ measureable PNe parallaxes down to $\mathrm{V}=16$. Despite the astonishing astrometric precision of GAIA, a long-baseline optical interferometer known as the SIM mission (due to launch in 2015) is designed to be yet more precise. The quoted wide-angle astrometric precision of $4 \mu$ as results in a $10 \sigma$ parallax for a PN at distances of $25 \mathrm{kpc}$. SIM is expected to have a faintness limit of $\mathrm{V}=20$. Unfortunately, the ability of SIM to generate huge numbers of PNe parallaxes is limited by being a pointed mission. Nevertheless, SIM will certainly impact the state of PNe trigonometric parallaxes. 


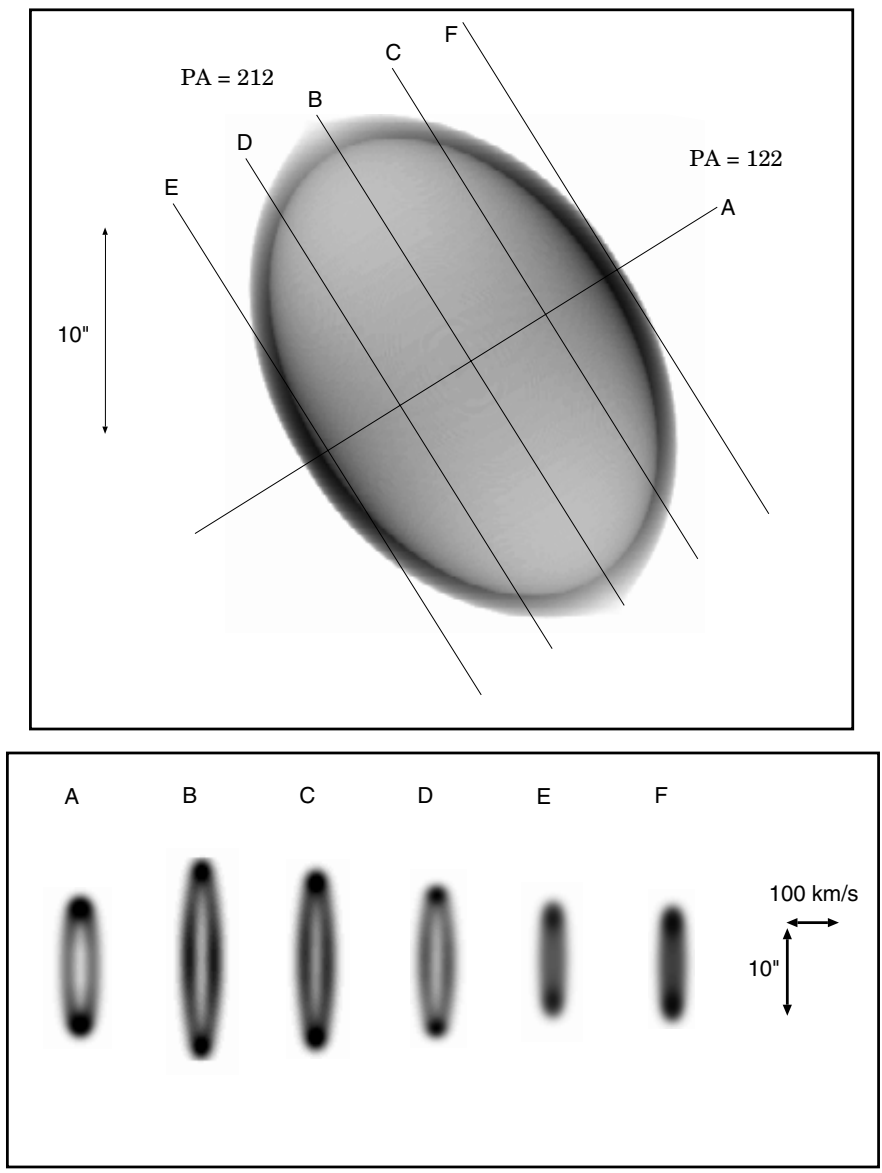

Figure 4. The modified-PES model of NGC 7354 in the light of [O III] that best matches the data shown in Figure 3 (in a least squares sense).

\section{Expansion Parallax}

The expansion parallax method is a technique that has been developed by many authors over the past decade (Masson 1986, 1989a, 1989b; Hajian, Terzian, \& Bignell 1993, 1995; Hajian \& Terzian 1996; Palen et al. 2002; and several others. See also Ruiz et al., this conference). The expansion parallax method was initially applied to images of the centimeter-wave free-free continuum obtained at the VLA. The high dynamic range and fidelity of the images combined with excellent angular resolution resulted in a set of PNe distances with errors as small as $25 \%$. However, the success of the expansion method was limited by the small $(<10)$ sample size of available targets.

The advent of high quality data from the Hubble Space Telescope reinvigorated expansion parallax research in PNe. Images of PNe from the WFPC2, in particular, have sufficient dynamic range and fidelity, and the systematic astrometric errors (especially the distortion) are well-enough understood, that high precision angular expansions can be measured from PNe with time baselines of a few years. To date, precision expansion parallax distances to a $<10$ PNe have been published (see Mellema [2004] for a recent summary). I will also be showing some preliminary results from an ongoing investigation (Hajian et al. 2006)below. 


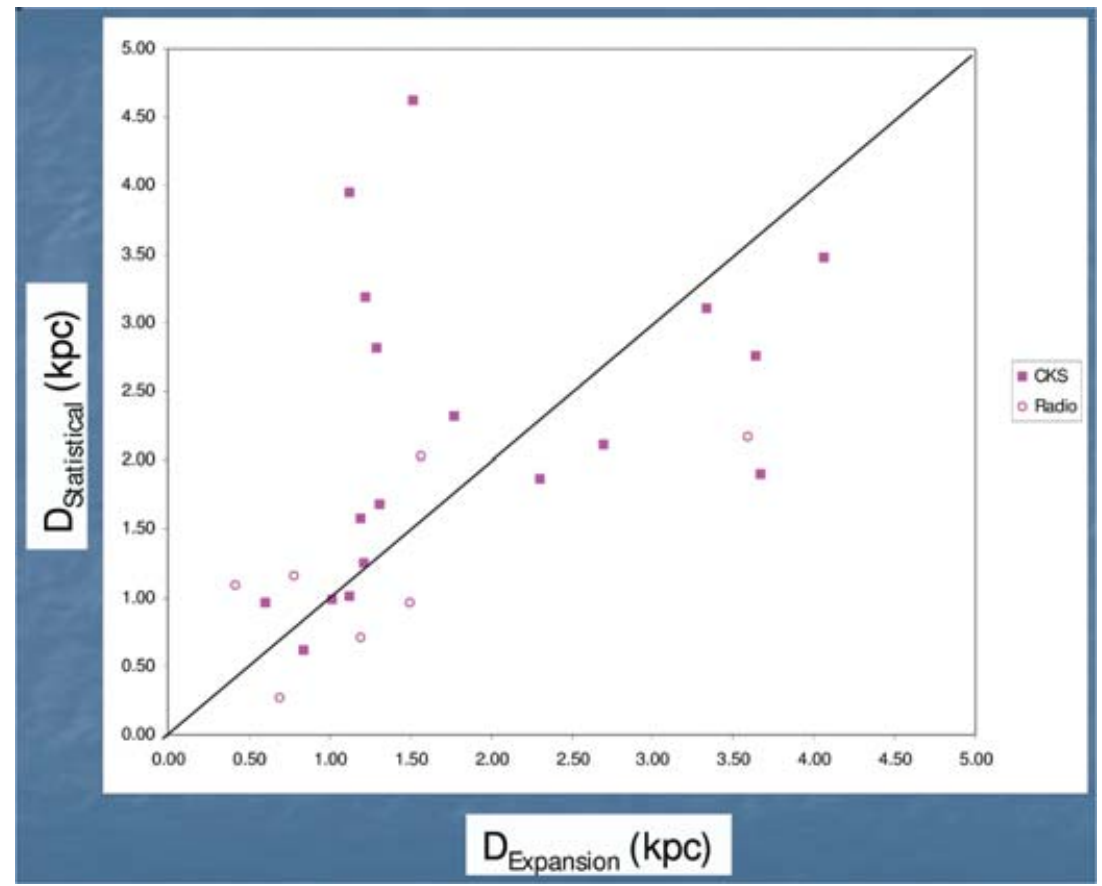

Figure 5. A comparison of Shklovsky distances (from the compilation of Cahn, Kaler, \& Stanghellini (1992) and the corresponding expansion parallax distances as computed by us (Hajian et al. 2006). The line through the origin with a slope of 1 is shown for reference. The $18 \mathrm{PNe}$ with optical expansion parallax distances from HST are shown as filled squares, while the 7 radio expansion parallax distances from the VLA are shown as open circles.

The basic recipe for the expansion parallax method is (1) Obtain the angular expansion rate. $\dot{\theta}$, of the PNe by analyzing two epochs of image data, and (2) Obtain the Doppler expansion velocity, $v_{\exp }$, of the $\mathrm{PN}$ with a spectrometer. The expansion parallax distance is:

$$
D=\left(\frac{v_{\exp }}{1 \mathrm{~km} / \mathrm{s}}\right)\left(\frac{1 \mathrm{mas} / \mathrm{yr}}{\dot{\theta}}\right)
$$

Two different techniques have been employed in order to measure the angular expansion rate of the PNe. They are known as the magnification and gradient methods. In the case of the magnification method, $\dot{\theta}$ is inferred by solving for the magnification factor, $M$, which is treated as a free parameter, based on two images separated by a time baseline of several years. For instance, $M=1$ implies that the PNe has not expanded between the two epochs, and that the angular expansion is not detectable. The goal is to find the value of $M$ which minimizes the residual difference between the second epoch image and an image consisting of the first epoch image magnified by $M$ relative to the nebular center (see Figure 1). The angular expansion of the nebula is therefore:

$$
\dot{\theta}=\frac{(M-1) \theta}{T}
$$

The advantage of the magnification method is that it is relatively fast. After the two images are aligned, the determination of a single parameter is not very painful. However, the magnification method fails if the $\mathrm{PN}$, or a significant portion of the $\mathrm{PN}$, is expanding non-homologously (e.g. when the nebula is interacting with the surrounding medium).

The other technique for determining $\dot{\theta}$ is the gradient method. This method is best envisioned for the case of a uniformly expanding ring. Subtracting the second epoch from 
the first epoch results in an image of the PN consisting of a negative ring bounding a positive ring. In Figure 2, I show an example of the nebular brightness, $F$, along a line passing through the central star of the PNe in one of the epoch images. The amplitude of the difference signature is $\Delta \mathrm{F}$. It is a simple matter to see that the angular expansion between epochs, $\Delta \theta$ is given by:

$$
\Delta \theta=\frac{\Delta F}{\nabla F}
$$

The gradient method is far more time-intensive than the magnification method. Consider the flux profiles along an individual rays passing through the nebular center. Each time the ray crosses bright, sharp nebulosity, a value of $\dot{\theta}$ can be estimated. This technique was fully explored in the case of the [O III] data from NGC 6543 (Reed et al. 1999), where the $\mathrm{PN}$ was subjected to 38 rays containing an average of 3 intersections with nebulosity each (see their Figure 2). However, the advantage of the gradient method is the direct result of the additional manpower required: the scalar field of radial $\mathrm{PNe}$ expansions is more accurately measured by the gradient method, and not limited to PNe exhibiting regular homologous evolution with time.

\subsection{Sources of Error}

There are several effects which can contribute significant systematic errors to the expansion parallax method. Some of these effects are inherent to any first-order treatment of nebular angular expansion. For example, these include the assumption that the nebular gas moves at constant velocity between epochs, that the nebular gas expands radially, etc. Although there are several theoretical arguments for such flows, a movie showing the temporal evolution of a nebular clump along an accelerated and/or non-radial trajectory has not yet been obtained.

A systematic error resulting from astrophysical considerations has been discussed in detail by several authors (e.g., Mellema 2004; Schoenberner, Jacob, \& Steffen 2005). At issue is whether the measured velocities correspond to the actual motion of the gas being imaged. If shocks are present, then the jump condition can be used to solve for a correction factor $(>1)$ which multiplies the observed Doppler velocity. As a result, PNe observed in the light of shocked lines must have their expansion parallax distances increased by a potentially significant factor. Space does not permit a thorough discussion of this worthwhile subject, although I will return to this subject at the end of this paper.

The description of the expansion parallax method above is fine for spherical, uniformly expanding PNe, but geometric modifications are required for a general PN. Even if the problem is restricted to prolate ellipsoidal geometries, the correction factor can be an order of magnitude larger than the random errors of the expansion parallax method. The following section describes recent progress in modeling and understanding the spatiokinematic structure of a sample of PNe.

\subsection{Recent Results}

Along with several of my collaborators, I initiated a program of expansion parallax measurement of a sample of PNe with the HST, supplemented by groundbased longslit spectroscopy at the $4 \mathrm{~m}$ telescopes at CTIO and KPNO. The spectroscopic slit was positioned at a variety of locations across the face of the nebula. The target list consists primarily of bright, nearby PNe with simple, elliptical morphologies, and sharp features.

In order to determine the nebular geometry and the structure of the velocity field, we adopted the Prolate Ellipsoidal Shell (PES, hereafter) model. Detailed explanations of the PES model are in the literature (Aaquist \& Kwok 1996; Zhang \& Kwok 1998). In summary, the PES model predicts the synthetic image of a gas distribution ionized 


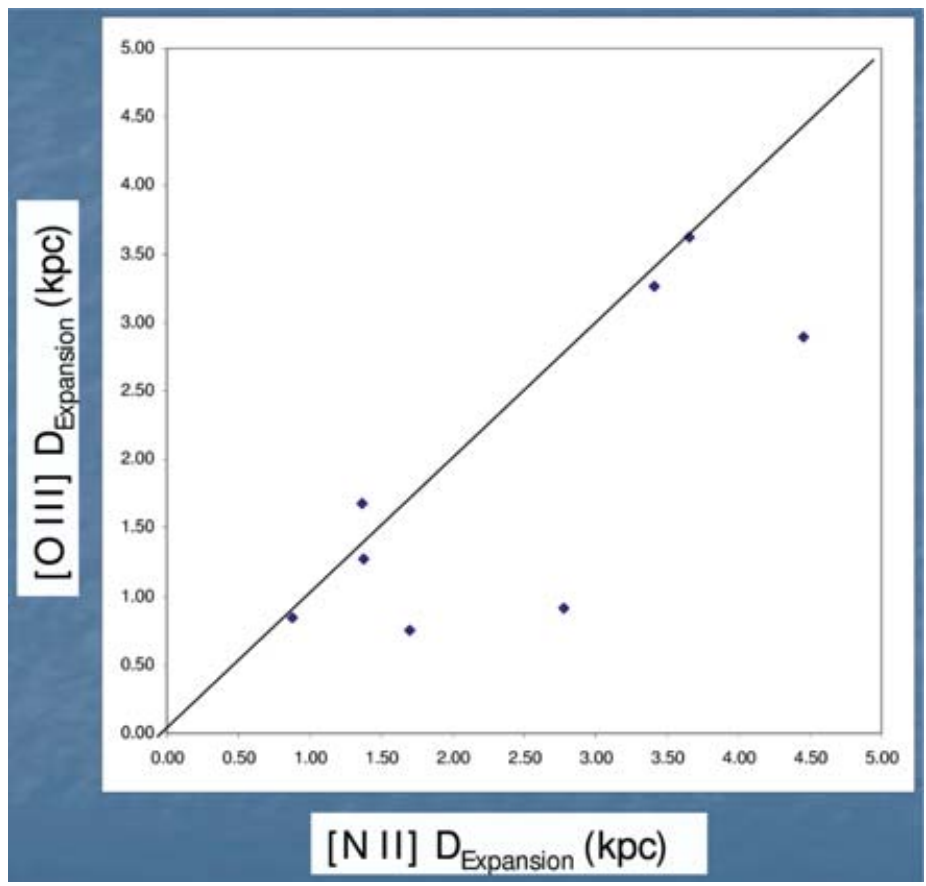

Figure 6. A comparison of the distances based on [N II] data and on [O III] data.

by a central source. The model contains ionization physics, and the gas is simply a parametrized distribution (i.e. there is no hydrodynamics in the model). The above references document the success of the PES model in reproducing the geometries of a wide range of $\mathrm{PNe}$. We felt that the model could be improved by including spectra (i.e. radial velocities), which we did by adding one parameter to the 12 in existence. The use of images and spectra to uniquely constrain nebular spatio-kinematic geometries is a powerful tool. The image data tends to constrain material perpendicular to the line-of-sight, while the spectral information tends to constrain nebulosity parallel to the line-of-sight. As an extreme example, a spherical nebula and a cigar-shaped nebula viewed along the major axis present very similar images, but the spectra will show substantial differences. An example of our application of the modified PES model is shown in Figures 3 and 4 for the [O III] data from and model for NGC 7354.

We derived angular expansion rates for the target PNe using the magnification method while focusing on the region near the equatorial rim (minor axis). This way, the equatorial expansion velocity, which is one of the parameters resulting from the modified-PES fits, can be divided by the equatorial angular expansion rate to result in a PN distance corrected for geometric effects (particularly the inclination of the major axis relative to the line-of-sight). The resulting expansion parallax distances are plotted in Figure 5 relative to the statistical distances from the compilation of Cahn, Kaler \& Stanghellini (1992). Although there is evident scatter about the line of unity slope, no clear trend is seen, even when the radio expansion parallax targets from the VLA are included. We conclude that the expansion distances seem consistent with these statistical distances.

Of special interest is the subsample of eight PNe with distances (independently) based on $[\mathrm{O}$ III $]$ and $[\mathrm{N} \mathrm{II}]$ observations. These data are plotted in Figure 6. The sample size is small, and so our conclusions are only suggestive, but if there is a trend in Figure 6, it is that the $[\mathrm{N}$ II] distances are slightly larger than the [O III] distances. This suggests 
that shock activity is not significant in the sampled PNe; shocks would result in an observed $[\mathrm{N}$ II] velocity smaller than the true gas velocity, and so application of the required correction factors will cause the $[\mathrm{N} \mathrm{II}]$ distances to further depart from the [O III] distances. A larger sample is necessary to increase the confidence of this conclusion.

\section{Conclusions}

Though the expansion parallax distances are expensive in terms of data and time, and yield individual distance estimates to $\mathrm{PNe}$ (i.e. one at a time), the total number of expansion parallax distances is growing and will soon exceed 30. Before long, a statistically significant sample will be achieved. There are still some sources of systematic error that may be present, the most notable being the potential effect of shock fronts altering the apparent kinematics of the nebular gas. The presence or absence of this phenomenon awaits a multi-ion expansion parallax study of individual PNe targets. Only by measuring the $\dot{\theta}$ and $v_{\text {exp }}$ for a series of ionic species spanning a range of shock sensitivity can the effect of shocks on expansion parallax distances finally be ascertained.

There will be a revolution in trigonometric parallaxes from future space astrometry missions. The simplicity of the trigonometric parallax makes the parallax distance a very attractive estimator, and the SIM and GAIA spacecrafts will be poised to increase the available sample of PNe parallax targets. In the meantime (10-15 years at least!), the precision $\mathrm{PNe}$ distance work being done will continue to expand the list of available targets while generating parallax calibrators for the upcoming space missions.

\section{Acknowledgements}

I would like to acknowledge the significant contributions of Bruce Balick, Andrew Cenko, Anne Jaskot, Tracy Klayton, Kevin Knuth, Steven Movit, Yervant Terzian, Denis Trofimov, and many others to this work.

\section{References}

Aaquist, O.B., \& Kwok, S. 1996, ApJ, 462, 813.

Benedict, G.F., et al., 2003, AJ, 126, 2549.

Cahn, J.H., Kaler, J.B., \& Stanghellini, L. 1992, A\&AS, 94, 399.

Hajian, A. R., Terzian, T. 1996, PASP, 108, 419.

Hajian, A.R., Terzian, Y., \& Bignell, C. 1993, AJ, 106, 1965.

Hajian, A.R., Movit, S.M., Trofimov, D., Balick, B., et al. 2006, ApJSS, submitted.

Harris, H.C., Dahn, C.C., Canzian, B., Guetter, H.H., et al. 2006, AJ, in preparation.

Masson, C.R. 1986, ApJ., 302, L27.

Masson, C.R. 1989a, ApJ., 336, 294.

Masson, C.R. 1989b, ApJ., 346, 243.

Mellema, G. 2004, A\&A, 416, 623.

Palen, S., Balick, B., Hajian, A.R., Terzian, Y., Bond, H., \& Panagia, N. 2002, AJ, 123, 2666.

Pottasch, S.R., \& Acker, A. 1998, A\&A, 329, L5.

Reed, D.S., Balick, B., Hajian, A.R., Klayton, T.L., et al. 1999, AJ, 118, 2430.

Schoenberner, D., Jacob, R., \& Steffen, M. 2005, A\&A, 441, 573.

Shklovsky, I.S. 1956, AZh, 33, 222.

Terzian, Y., \& Teymourian, A. 2005, in The Initial Mass Function 50 Years Later, eds.

E. Corbelli, F. Palla, \& H. Zinnecker (Springer: Dordrecht), pp. 521-526.

Zhang, C.Y., \& Kwok, S. 1998, ApJS, 117, 341. 